\title{
The risk stratification in patients with heart failure: the value of considering both global longitudinal left ventricular strain and mechanical dispersion
}

\begin{tabular}{|c|c|}
\hline Journal: & Canadian Journal of Physiology and Pharmacology \\
\hline Manuscript ID & cjpp-2017-0049.R1 \\
\hline Manuscript Type: & Article \\
\hline Date Submitted by the Author: & 14-Apr-2017 \\
\hline Complete List of Authors: & $\begin{array}{l}\text { Mornoş, Cristian; "Victor Babes" University of Medicine and Pharmacy, } \\
\text { Cardiology } \\
\text { Muntean, Danina; University of Medicine and Pharmacy Timisoara, } \\
\text { Pathophysiology } \\
\text { Mornos, Aniko; Institute of Cardiovascular Diseases, Timişoara, Romania } \\
\text { Crisan, Simina; "Victor Babes" University of Medicine and Pharmacy, } \\
\text { Timişoara, } \\
\text { Petrescu, Lucian; "Victor Babes" University of Medicine and Pharmacy, } \\
\text { Timişoara, } \\
\text { Ionac, Adina; "Victor Babes" University of Medicine and } \\
\text { Pharmacy, Timisoara, } \\
\text { Sosdean, Raluca; "Victor Babes" University of Medicine and Pharmacy, } \\
\text { Timişoara, } \\
\text { Cozma, Dragos; "Victor Babes" University of Medicine and Pharmacy, } \\
\text { Timişoara, }\end{array}$ \\
\hline $\begin{array}{r}\text { Is the invited manuscript for } \\
\text { consideration in a Special } \\
\text { Issue?: }\end{array}$ & IACS European Section 2016 \\
\hline Keyword: & $\begin{array}{l}\text { heart failure, malignant ventricular arrhythmia, cardiovascular risk } \\
\text { stratification, global longitudinal strain, mechanical dispersion }\end{array}$ \\
\hline
\end{tabular}

\section{SCHOLARONE" \\ Manuscripts}


The risk stratification in patients with heart failure: the value of considering both global longitudinal left ventricular strain and mechanical dispersion

Authors:

Cristian Mornoş, ${ }^{\mathrm{a}, \mathrm{b}}$, Danina Muntean ${ }^{\mathrm{a}}$, Aniko Mornoş ${ }^{\mathrm{b}}, \operatorname{Simina}_{\text {Crişan }}^{\mathrm{a}}{ }^{\mathrm{a}}$ Lucian Petrescu ${ }^{\mathrm{a}, \mathrm{b}}$, Adina Ionac $^{\mathrm{a}, \mathrm{b}}$, Raluca Sosdean ${ }^{\mathrm{a}}$, Dragoş Cozma ${ }^{\mathrm{a}, \mathrm{b}}$,

a "Victor Babes" University of Medicine and Pharmacy, Timişoara, Romania

${ }^{\mathrm{b}}$ Institute of Cardiovascular Diseases, Timişoara, Romania

Correspondence to:

Lucian Petrescu

"Victor Babes" University of Medicine and Pharmacy, Cardiology Clinic

Timişoara, G. Adam 13A

Romania

mornoscristi@gmail.com

tel: +40256207355

fax: +40256207362 
In previous studies mechanical dispersion (MD) predicted ventricular arrhythmias independently of left ventricular ejection fraction (LVEF). Moreover the combination of MD and global longitudinal strain (GLS) increased the prediction of arrhythmic events. We investigated the prognostic value of a new 2D-strain index, GLS/MD, in patients with heart failure (HF). We analysed 340 consecutive HF outpatients in sinus rhythm. Echocardiography was performed at 1.6 \pm 0.4 months after hospital discharge. The end point included sudden cardiac death, ventricular fibrillation and sustained ventricular tachycardia (SCD/VA). During the follow-up period (36 \pm 9 months) SCD/VA occurred in 48 patients (14.1\%). By using the multivariate Cox regression analysis, including $\mathrm{LVEF}$, early diastolic transmitral/mitral annular velocity ratio (E/E'), GLS, MD and GLS/MD in the model, GLS/MD was the best independent predictor of $\mathrm{SCD} / \mathrm{VA}(\mathrm{HR}=3.22,95 \%$ confidence interval $=1.72-6.15, p=0.03)$. Separate inclusion of LVEF, systolic mitral annular velocity, E/E', GLS and MD together with GLS/MD, showed that GLS/MD remained the best predictor of SCD/VA (each $p<0.05$ ). The optimal GLS/MD cut-off value to predict SCA/VA was $-0.20 \% / \mathrm{ms}$ ( $80 \%$ sensitivity, $76 \%$ specificity). Irrespective of LVEF, free survival was significantly better in patients with GLS/MD $\leq-0.2 \% / \mathrm{ms}$ (log-rank, $p<0.001)$. In conclusion, GLS/MD may improve cardiovascular risk stratification in subjects with HF.

Keywords: heart failure, malignant ventricular arrhythmia, cardiovascular risk stratification, 2Dstrain imaging, global longitudinal strain, mechanical dispersion, GLS/MD ratio 


\section{Introduction}

Cardiac imaging is indispensable for heart failure (HF) patients' management. Prognosis in cardiac disease is closely related to systolic function which is commonly assessed using left ventricular ejection fraction (LVEF). Two-dimensional strain echocardiography may potentially save additional lives using a more appropriate risk stratification of patients with HF. An increasing number of studies have suggested that LV global longitudinal strain (GLS) is a robust, well validated and reproducible marker for the measurement of LV longitudinal deformation, which is superior to EF, both as a measure of LV function and as well as a predictor of mortality and cardiac events (Cho et al. 2009; Ersbøll et al. 2013a; Huagaa et al. 2012; Iacoviello et al. 2013; Kalam et al. 2014; Mignot et al. 2010; Russo et al. 2014; Smiseth et al. 2016; Stanton et al. 2009). A cutoff value for GLS as a marker of high mortality risk is not available at the moment (Smiseth et al. 2016). The lack of standardization across commercially available 2D-strain software makes the evaluation of myocardial strain vendor-dependent (Russo et al. 2014).

Mechanical dispersion (MD), reflecting contraction heterogeneity, is a novel riskstratifying parameter derived also from 2D-strain imaging. This parameter proved to be an excellent predictor of arrhythmic events independently of LVEF (Smiseth et al. 2016). MD is calculated as the standard deviation of contraction duration measured from the electrocardiographic R-wave to peak shortening strain in multiple LV segments (Huagaa et al. 2013a). Normally, all segments have relatively similar contraction duration and therefore low values for MD. MD can be regarded as the mechanical consequence of electrical alterations and tissue abnormalities (Huagaa et al. 2010a). MD quantification is an accurate parameter in predicting mortality and ventricular arrhythmias in patients with previous myocardial infarction (Huagaa et al. 2010b, Huagaa et al. 2013a), non-ischaemic cardiomyopathy (Huagaa et al. 2012), 
HF and cardiac resynchronization therapy (CRT) (Hasselberg et al. 2016). MD may be used as an additive parameter for risk assessment in these populations.

Recently, Huagaa et al. suggested that a combination of MD and global strain may improve the selection of patients with previous myocardial infarction for implantable cardioverter-defibrillator therapy (ICD) (Huagaa et al. 2013a). Therefore we proposed a new 2Dstrain parameter (GLS/MD) and we tested the hypothesis that it might improve the risk stratification of major ventricular arrhythmic events in patients with HF.

\section{Methods}

\section{Patients}

Four hundred and sixteen consecutive outpatients (age $\geq 18$ years) suffering from chronic HF (both with and without reduced HF) with sinus rhythm and optimal medical treatment were prospectively analyzed. All patients were previously hospitalized in Timisoara Institute of Cardiovascular Diseases for exacerbation of HF symptoms with at least 1 New York Heart Association class deterioration, typical signs of HF and echocardiographic evidence of systolic and/or diastolic LV dysfunction (McMurray et al. 2012). Patients with non-cardiac disease with a life expectancy $<1$ year, inadequate echocardiographic images, significant primary valvular heart disease, acute coronary syndrome in the previous 40 days, coronary artery by-pass graft before echocardiographic examination, congenital heart disease, and patients undergoing ventricular pacing were excluded. The study was conducted in full conformity with the current revision of the Declaration of Helsinki. The study was approved by the local research ethics committee and informed consent was obtained from all individual participants included in the study. 


\section{Echocardiography}

Echocardiography was performed at $>1$ month of hospital discharge with an ultrasonographic system (Vivid 9 General Electric, Milwaukee, WI) and analyzed using a commercially available software (EchoPAC; GE Vingmed Ultrasound AS). LVEF was assessed from apical 2- and 4-chamber views using a modified Simpson's rule (Lang et al. 2006). Left atrial (LA) volume was calculated using the biplane area-length method in the apical fourchamber and apical two-chamber views at ventricular end-systole (maximum LA size) (Lang et al. 2006). The severity of mitral regurgitation was assessed from the apical views using proximal convergence method; the regurgitant orifice area (ROA) and the regurgitant volume (RV) were determined (Zoghbi et al. 2003). The peak early diastolic velocity of transmitral flow (E) was measured by pulsed-wave Doppler during end-expiratory apnea. Measurement of systolic pulmonary artery pressure (SPAP) was performed using the maximal regurgitant velocity at the tricuspid valve by continuous wave Doppler. The peak early diastolic (E') and peak systolic velocities $\left(\mathrm{S}^{\prime}\right)$ were determined at the lateral and septal mitral annulus corners using pulsed wave Tissue Doppler Imaging (TDI) (Nagueh et al. 2009). The average value of the velocities was used for the analysis and E/E' was calculated.

GLS was determined from apical planes (4-, 3-, and 2-chamber view) using 2D-strain echocardiography. Three cardiac cycles from each view were recorded. Aortic valve closure was identified on continuous-wave Doppler recording through the aortic valve. Peak systolic strain was assessed in all LV segments and the values from each segment were averaged to obtain GLS (Reisner et al. 2004). If more than 3 of the LV segments were inadequately tracked, the patient was excluded from the final analysis. MD was defined as the standard deviation of time measured from the peak R-wave to peak negative strain in multiple LV segments (Huagaa et al. 
2010a). Segments exhibiting only positive strain values, like dyskinetic segments, as well as segments with strain curves oscillating around the zero line, like akinetic segments, were not included in time measurements (Huagaa et al. 2013a). If 6 or more segments did not have sufficient tracking, the patient was excluded from the analyses. GLS/MD was calculated (Figure 1).

Echocardiographic analyses were performed offline by 2 experienced sonographers (M.C. and C.D.) blinded to clinical data. The inter- and intra-observer variabilities for GLS, MD and GLS/MD were examined. Measurements were performed in a group of 30 randomly selected subjects by one observer two separate times and by two investigators who were unaware of one another's measurements and of the study time point.

\section{Clinical Variables Recorded}

The following clinical variables were recorded: age, sex, body mass index, mean arterial pressures, heart rate, etiology of HF, NYHA functional class. Prescription of the main therapeutic classes in HF was also noted.

\section{Clinical Outcome}

Patients were followed-up for $\geq 24$ months. Documented sustained ventricular tachycardia, ventricular fibrillation, and sudden cardiac death were regarded as the study endpoint (SCD/VA). Cardiovascular events were assessed from hospital documentation, information from attending physicians and death certificate. No patient was lost to follow-up. 


\section{Statistical Analysis}

Data are expressed as mean \pm standard deviation for continuous variables and as proportions for categorical variables. Continuous variables were compared between groups using unpaired $t$ test (variables with normal distribution) or Mann-Whitney $U$ test (non-normally distributed variables). Proportions were compared using chi-square test and Fischer's exact test. Univariate Cox proportional hazards analysis was performed to investigate the significance of a number of variables in predicting SCD/VA. Some variables associated with outcome were included into a multivariate Cox regression model to identify independent predictors of $\mathrm{SCD} / \mathrm{VA}$. The output of this analysis was expressed as hazard ratio with $95 \%$ confidence interval. Cumulative events curves were obtained using the Kaplan-Meier method. Patients who died of non-arrhythmic causes were censored (as non-events) at date of death. A $p$ value $<0.05$ was considered significant. Receiver-operator characteristic (ROC) curves were plotted to define cut-off values of independent predictors. Intra- and inter $\square$ observer variability for GLS, MD and GLS/MD were measured by the intraclass correlation coefficient and by the coefficient of variation $(\mathrm{CV})$ with the root-mean-square method. All analyses were carried out with the SPSS, version 18.0 (SPSS Inc., Chicago, Illinois) statistical software.

\section{Results}

\section{Baseline characteristics and arrhythmic events}


The current prospectively study included 340 consecutive outpatients $(63 \pm 12$ years; 111 women), previously hospitalized for HF, irrespective of LVEF. The mean LVEF was $42 \pm 13 \%$ and the aetiology of HF was represented by coronary artery disease (215 patients), non-ischemic cardiomyopathy (87 patients) and systemic hypertension (38 patients). Table 1 presents the baseline characteristics of the 340 patients included in the study.

During the $36 \pm 9$ months of follow-up, SCD/VA occurred in 48 patients (14.1\%). Of these 48 patients, 2 received appropiate schock therapy for ventricular fibrillation from an ICD implanted for primary prevention, 9 had documented ventricular tachycardia requiring cardioversion and 37 died suddenly. Four of the patients requiring cardioversion died during the same hospitalization.

\section{Echocardiographic parameters as markers of arrhythmic risk}

Echocardiography was performed at the moment of inclusion in the study $(1.6 \pm 0.4$ months after hospital discharge). Of the initial 416 patients, 65 were excluded because of suboptimal image quality for speckle-tracking analysis and 11 because of event occurrence before being enrolled in the study. The echocardiographic characteristics of the remaining 340 patients with and without arrhythmic events are presented in Table 2. Eight of the 48 SCD/VA patients subgroup (16.7\%) already had an ICD implanted compared to only 27 of $292(9.3 \%)$ in the non-SCD/VA subgroup $(p<0.001)$.

Patients who developed SCD/VA had significantly higher SPAP, larger LA and LV volumes, lower LVEF and S' velocities, and higher values for E/E', MD, GLS and GLS/MD as compared with patients without SCD/VA. In addition, there was no difference regarding the 
distribution of ROA and RV. Mean GLS/MD ratio was $-0.24 \pm 0.31 \% / \mathrm{ms}$ in patients who developed SCD/VA and $-0.83 \pm 0.54 \% / \mathrm{ms}$ in the rest $(p<0.001)$.

Table 3 shows the echocardiographic variables that predicted SCD/VA on univariate Cox regression analysis $(p<0.05)$ : LA volume, LV end-diastolic volume, LV end-systolic volume, LVEF, SPAP, S', E/E', GLS, MD and GLS/MD. By including LVEF, E/E', GLS, MD and GLS/MD ratio in the model, the multivariate forward Cox regression analysis (Table 3) identified GLS/MD as the best independent predictor of SCD/VA (HR=3.22, 95\% confidence interval $=1.72-6.15, p=0.03)$, while $\mathrm{E} / \mathrm{E}$ ' ratio also remained a good predictor $(\mathrm{HR}=1.14,95 \%$ confidence interval=1.08-1.22, $p=0.008)$. Including GLS/MD together with $\mathrm{E} / \mathrm{E}$, only GLS/MD turned out to be a better predictor $(\mathrm{HR}=2.4, p=0.003)$ than $\mathrm{E} / \mathrm{E}^{\prime} \quad(\mathrm{HR}=1.12, p=0.001)$. Furthermore, separate inclusion of LVEF, S', GLS and MD (all $p>0.05$ ) showed that only GLS/MD predicted SCD/VA (all $p<0.01$ ). The additional benefit of GLS/MD to predict $\mathrm{SCD} / \mathrm{VA}$ is shown in Figure 2. However, the addition of GLS/MD markedly improved the prognostic utility of the model containing LVEF, S', E/E', GLS and MD $(p=0.001)$.

Figure 3 shows the ROC curve for GLS/MD to predict SCD/VA. The optimal cut-off value was $-0.20 \% / \mathrm{ms}$ with $80 \%$ sensitivity and $76 \%$ specificity. Arrhythmia-free survival was significantly better in those with GLS/MD $\leq-0.2 \% / \mathrm{ms}$ (log-rank, $p<0.001)$ as shown by the Kaplan-Meier analysis (Figure 4a). The median event-free survival time from the baseline echocardiography was 40.3 months in the group of patients with GLS/MD $\leq-0.20 \% / m s(193$ patients, 56.8\%) and 29.8 months in those with GLS/MD >-0.20\%/ms (147 patients, 43.2\%).

\section{HF with LVEF $\leq 35 \%$ versus HF with $L V E F>35 \%$}


To investigate the possible impact of LVEF, patients with LVEF $\leq 35 \%$ (123 patients, $36.2 \%)$ and with LVEF $>35 \%$ (217 patients, 63.8\%) were analyzed separately. The echocardiographic findings of this patients are presented in Table 4. In both groups, E/E', S', GLS, MD and GLS/MD were more altered in those with SCD/VA. The event-free survival rate was significantly higher in patients with GLS/MD $\leq-0.2 \% / \mathrm{ms}$ (log-rank, $p<0.001)$ regardless of LVEF ( $\leq 35 \%$ or $>35 \%$ ), as shown by the Kaplan-Meier plots (Figure $4 \mathrm{~b}$ and $4 \mathrm{c}$ ).

\section{Reliability and feasibility}

The intra-observer intraclass coefficients for GLS, MD and GLS/MD were 0.93 (CV 2.7\%), $0.91(\mathrm{CV} \mathrm{3.1 \% ),} \mathrm{and} 0.91$ ( $\mathrm{CV} \mathrm{2.9 \% ),} \mathrm{respectively.} \mathrm{The} \mathrm{inter-observer} \mathrm{intraclass}$ coefficients for GLS, MD’ and GLS/MD were 0.92 (CV 2.8\%), 0.90 (CV 3.1\%), and 0.91 (CV $3.1 \%$ ), respectively.

\section{Discussion}

To the best of our knowledge this is the first study investigating the value of a new 2Dstrain derived index, GLS/MD, to predict major ventricular arrhythmic events in patients with $\mathrm{HF}$, in sinus rhythm. In our work, GLS/MD ratio was the strongest predictor of SCD/VA when compared to other echocardiographic parameters (LVEF, E/E', S', GLS or MD).

The clinical importance of predicting arrhythmic events in patients with or without LV dysfunction is crucial. Previous studies with echocardiographic imaging have suggested that LVEF (Nikitin et al. 2006), LV volumes indices (Nikitin et al. 2006) and LA size (Lim et al. 2009; Rosca et al. 2011) are good predictors of outcome in the setting of congestive HF. 
Currently, LVEF alone is used to assess the risk of ventricular arrhythmia and indication for primary prevention ICD in HF patients (Hasselberg et al. 2016; McMurray et al. 2012). LVEF is an obvious marker of prognosis, with a cut off value $<35 \%$ indicating poor prognosis, but there is criticism and debate around it (Buxton et al. 2010; Huagaa et al. 2010a; McMurray et al. 2012). On the other hand, remodeling in smaller regions of the LV may be missed by LVEF (Huagaa et al. 2013a). Moreover, in our study, LVEF, predictor of SCD/VA on univariate analysis, was eliminated on multivariate analysis.

Unlike LV volumes and LVEF, TDI does not require tracing of endocardial contours (Nikitin et al. 2006). The E/E' ratio is an index that reflects accurately the LV filling pressure (Nagueh et al. 2009), and it is a strong predictive factor for survival. Møller et al. (2006) studied a group of patients after first myocardial infarction and reported that $E / E$ ' was an independent predictor of all-cause death. Other studies (Almeida et al. 2012; Hirata et al. 2009; Olson et al. 2008) reported that $\mathrm{E} / \mathrm{E}$ ' ratio and $\mathrm{S}$ ' wave (Nikitin et al. 2006) were strong independent predictors of outcome in populations with HF, while $S$ ' was a predictor of cardiac mortality in heterogeneous populations of cardiac patients (Mogelvang et al. 2015; Wang et al. 2003). Changes in E' precede the changes in atrial size and atrial enlargement may be a result of longstanding diastolic changes (Huagaa et al. 2013b). A decreased E' value may reflect regional differences in onset of myocardial relaxation resulting from heterogeneity in contraction duration (Huagaa et al. 2009). E' is associated with an intense energy demanding process, influenced by myocardial ischemia, while $\mathrm{S}^{\prime}$ wave indicates myocardial deterioration with increased LV stiffness and consecutive systolic dysfunction (Mogelvang et al. 2015). Nevertheless, TDI has technological limitations such as angle dependence, signal noise, and measurement variability. 
Furthermore, the current study demonstrated that in our group of HF patients, GLS/MD predicts $\mathrm{SCD} / \mathrm{VA}$ better than $\mathrm{S}^{\prime}$ or $\mathrm{E} / \mathrm{E}$ '.

Two-dimensional strain is not angle-dependent and GLS has proved to be able to detect early LV systolic dysfunction in a variety of conditions, even when LVEF is still in the normal range (Russo et al. 2014). It is thought that longitudinal contraction is a particular marker of subendocardial function, which may be disproportionately involved in subclinical disease, including hemodynamic overload, subendocardial ischemia, or early myocardial damage at a stage when LVEF is not yet impaired (Ersbøll et al. 2013a; Russo et al. 2014; Stanton et al 2009). GLS correlates with the infarct size and predicts death and HF after acute myocardial infarction as well as cardiovascular outcome (Ersbøll et al. 2013b). Several studies have examined the prognostic value of GLS in unselected populations (Stanton et al. 2009), in stable chronic HF (Cho et al. 2009; Mignot et al. 2010; Nahum et al. 2010), and recently in patients with chronic ischemic cardiomyopathy (Bertini et al. 2012). In HF patients fulfilling CRT indications, GLS before CRT implantation was an important predictor of survival free from death and heart transplantation (Hasselberg et al. 2016). The geometric alignment of the endocardial longitudinal fibers results in a smaller curvature and thus exposes them to greater detrimental effect of increased LV cavity pressure because of the law of Laplace (Ersbøll et al. 2013a). Our findings are consistent with previous studies showing that GLS can detect subtle myocardial changes of prognostic importance in patients with HF and improve arrhythmic risk stratification.

$\mathrm{MD}$, a new parameter derived from 2D-strain imaging, reflects contraction heterogeneity and was an excellent predictor of arrhythmic events independently of LVEF (Huagaa et al. 2013a). Potential etiologies of MD are electrical alterations, fibrosis or ischemia which may 
cause local delays in electromechanical activation. Furthermore, non-uniform loading conditions in a diseased ventricle may impacts the timing of peak shortening. Previous works have shown strong evidence that MD predicts ventricular arrhythmia in patients with previous myocardial infarction (Huagaa et al. 2010b; Huagaa et al. 2013a), non-ischaemic cardiomyopathy (Huagaa et al. 2012), and with HF and CRT (Hasselberg et al. 2016). Mismatch between electrical and mechanical systole has previously been suggested as a mechanism for ventricular arrhythmias (ter Bekke and Volders. 2012). MD remained a marker of arrhythmias even when excluding patients with wide QRS intervals (Huagaa et al. 2013a). Our findings are in accordance with the previous work of Ersbøll M et al. (2013b) by analyzing patients with acute myocardial infarction where both GLS and MD were significantly and independently related to ventricular arrhythmic events. In our study, adding MD to a model already including GLS did not significantly improve the risk assessment and MD was a poorer predictor than GLS/MD.

The recent development of 2D-strain imaging has facilitated the assessment of long-axis function, which is sensitive to the preclinical phases of $\mathrm{HF}$, as well as the evaluation of LV synchrony. Ventricular arrhythmias and sudden cardiac death in patients with chronic ischemic HF have been shown to be closely related to the extent of myocardial scarring and scar tissue heterogeneity assessed by cardiac magnetic resonance (Ersbøll et al. 2013). It makes no sense to just look at one of the 2D-strain parameters, since they may all provide different important information. Our finding is that combining the information from GLS and MD provides additional value to established arrhythmic risk factors. The addition of GLS/MD markedly improved the prognostic utility of the model containing LVEF, S', E/E', GLS and MD.

Three-dimensional (3D) strain imaging was developed as a new application that takes advantage of pyramidal and strain data that includes the whole LV and that can improve 
information about LV segmental and global deformation by avoiding the loss of speckles encountered in monoplane 2D-strain analysis. 3D-strain imaging offers the advantage of simultaneously measuring longitudinal, radial and circumferential strain values in the whole LV myocardium (Rosa et al. 2016). Area strain, a new parameter that reflects the global function assessment of the endocardial myocardium, is uniquely provided by $3 \mathrm{D}$-strain. It represents the sum of longitudinal and circumferential strain vectors. In addition, 3D-strain has been shown to improve reproducibility and accuracy and may be more useful than 2D techniques. Unfortunately, 3D-strain imaging has a limitation of temporal resolution and a vendor-dependent variability of deformation data. However, it holds great promise as a reliable and feasible tool for evaluating both overall and regional myocardial function (Seo et al. 2014).

CRT has proven to be highly effective for improving symptoms and survival of patients with advanced HF and wide QRS. Multicenter studies suggest that the predictive value of echocardiography was not sufficiently robust to replace routine selection criteria for CRT. The PROSPECT trial analyzing 12 echocardiographic parameters of dyssynchrony found that no single parameter could reliably improve patient selection for CRT; nevertheless, in this trial there was a wide variability in the echocardiographic measures' analysis (Chung et al. 2008). A more recent approach using speckle-tracking echocardiography yielded more accurate quantification of regional wall contraction and therefore generated a great deal of interest about their clinical applications for CRT. Strain imaging can play an important supplementary part in CRT on the basis of a case-by-case clinical decision for challenging cases (Khan et al. 2016). Patients undergoing CRT (with high risk of SCD/VA) were not included in our study group.

\section{HF with LV $>35 \%$}


The reduction in mortality by CRT in HF is well documented (McMurray et al. 2012). Currently, EF is used to assess risk of ventricular arrhythmia and indication for primary prevention ICD in HF patients. Although the absolute risk for SCD is highest in patients with significantly reduced LVEF, a significant proportion of SCD events occur in patients with LVEF $>35 \%$, which underscores the need for improved risk stratification after myocardial infarction (Ersbøll et al. 2013b). Despite the small number of events in our study, we found that GLS/MD was a marker of ventricular arrhythmic events in patients with LVEF $>35 \%$. These results indicate that GLS/MD may be a more accurate marker to detect small changes in myocardial function, which may be important in arrhythmic risk stratification. Haugaa et al. (2013a) demonstrate that after myocardial infarction, MD predicted arrhythmic events independently of LVEF and was an excellent predictor also in patients with preserved LV function. Also Ersbøll et al. (2013a) showed that GLS provides independent information related to death or HF admissions in patients with LVEF $>40 \%$ over and above well-established risk factors that carry treatment recommendations in current guidelines. In our study GLS/MD showed an independent significant association with incident ventricular events even among subjects with LVEF $>35 \%$, and its prognostic value is important to evaluate patients with arrhythmic risk who do not fulfill current ICD indications. The event-free survival rate was significantly higher in the group of patients with $\mathrm{GLS} / \mathrm{MD} \leq-0.20 \% / \mathrm{ms}$ regardless of $\mathrm{LVEF}(\leq 35 \%$ or $>35 \%)$.

\section{Limitations}

Our results should be considered in the context of several limitations. The number of patients in this study was relatively small. In our study, 2 independent observers performed strain analyses, with excellent intra- and inter-observer reliability. Unfortunately, $18 \%$ of patients were 
excluded from the initial study group (65 patients presented suboptimal image quality for speckle-tracking analysis) which is a potential bias. In addition, the study center functioned as a tertiary invasive center and therefore the study population may not reflect a general population of patients with HF. However, we were able to reach several significant observations. Coronary artery disease was highly prevalent in the present series and one cannot rule out the occurrence of ischemic events contributing to the sudden death of the patients. Also, the fact that our study sample was predominantly Caucasian limits the generalizability of our findings. Our study is a single-center study and its reproduction in other centers or by multicenter studies would argue for its validity.

\section{Conclusions}

In patients with HF, in sinus rhythm, the novel GLS/MD index is an important independent predictor of malignant ventricular events. GLS/MD may improve cardiovascular risk stratification in subjects with $\mathrm{HF}$, irrespective of LVEF value. GLS/MD ratio may become the optimal method for assessment of SCD/VA risk stratification.

\section{Acknowledgments}

This study was supported by the University Grant no. PIII-C1-PCFI-2014/2015-04.

Conflict of interest: none declared. 


\section{References}

Almeida, P., Rodrigues, J., Lourenço, P., Julia Maciel, M., and Bettencourt, P. 2012. Prognostic Significance of Applying the European Society of Cardiology Consensus Algorithm for Heart Failure With Preserved Systolic Function Diagnosis. Clin. Cardiol. 35(12):770-776. doi: 10.1002/clc.22044. PMID: 22886726.

Bertini, M., Ng, A.C., Antoni, M.L., Nucifora, G., Ewe, S.H., Auger, D., et al. 2012. Global longitudinal strain predicts long-term survival in patients with chronic ischemic cardiomyopathy.

Circ. Cardiovasc. Imaging, 5:383-391. doi: 10.1161/CIRCIMAGING.111.970434. PMID: 22412068.

Buxton, A.E., Ellison, K.E., Lorvidhaya, P., and Ziv, O. 2010. Left ventricular ejection fraction for sudden death risk stratification and guiding implantable cardioverter defibrillators implantation. J. Cardiovasc. Pharmacol. 55:450-455. PMID: 20509178.

Cho, G-Y., Marwick, T.H., Kim, H-S., Kim, M-K., Hong, K-S., and Oh, D-J. 2009. Global 2dimensional strain as a new prognosticator in patients with heart failure. J. Am. Coll. Cardiol. 54:618-624. doi: 10.1016/j.jacc.2009.04.061. PMID: 19660692.

Chung, E.S., Leon, A.R., Tavazzi, L., Sun, J.P., Nihoyannopoulos, P., Merlino, J., et al. 2008.

Results of the predictors of response to CRT (PROSPECT) trial. Circulation, 117:2608-2616. doi:10.1161/CIRCULATIONAHA.107.743120. PMID: 18458170.

Ersbøll, M., Valeur, N., Mogensen, U.M., Andersen, M.J., Moller, J.E., Velazquez, E.J., et al. 2013a. Prediction of all-cause mortality and heart failure admissions from global left ventricular longitudinal strain in patients with acute myocardial infarction and preserved left ventricular ejection fraction. J. Am. Coll. Cardiol. 61:2365-2373. doi: 10.1016/j.jacc.2013.02.061. PMID: 23563128. 
Ersbøll, M., Valeur, N., Andersen, M.J., Mogensen, U.M., Vinther, M., Svendsen, J.H., et al. 2013b. Early echocardiographic deformation analysis for the prediction of sudden cardiac death and life-threatening arrhythmias after myocardial infarction. JACC Cardiovasc. Imaging, 6(8):851-860. doi: 10.1016/j.jcmg.2013.05.009. PMID: 23850252.

Hasselberg, N.E., Haugaa, K.H., Bernard, A., Ribe, M.P., Kongsgaard, E., Donal, E., et al. 2016. Left ventricular markers of mortality and ventricular arrhythmias in heart failure patients with cardiac resynchronization therapy. Eur. Heart J. Cardiovasc. Imaging, 17(3):343-350. doi: 10.1093/ehjci/jev173. PMID: 26164406.

Haugaa, K.H., Edvardsen, T., Leren, T.P., Gran, J.M., Smiseth, O.A., and Amlie, J.P. 2009. Left ventricular mechanical dispersion by tissue Doppler imaging: a novel approach for identifying high-risk individuals with long QT syndrome. Eur. Heart J. 30:330-337. doi: 10.1093/eurheartj/ehn466. PMID: 18940888.

Haugaa, K.H., Amlie, J.P., Berge, K.E., Leren, T.P., Smiseth, O.A., and Edvardsen, T. $2010 a$. Transmural differences in myocardial contraction in long-QT syndrome: mechanical consequences of ion channel dysfunction. Circulation, 122:1355-1363. doi: 10.1161/CIRCULATIONAHA.110.960377. PMID: 20855658.

Haugaa, K.H., Smedsrud, M.K., Steen, T., Kongsgaard, E., Loennechen, J.P., Skjaerpe, T., et al. 2010b. Mechanical dispersion assessed by myocardial strain in patients after myocardial infarction for risk prediction of ventricular arrhythmia. JACC Cardiovasc. Imaging, 3:247-256. doi: 10.1016/j.jcmg.2009.11.012. PMID: 20223421.

Haugaa, K.H., Goebel, B., Dahlslett, T., Meyer, K., Jung, C., Lauten, A., et al. 2012. Risk assessment of ventricular arrhythmias in patients with nonischemic dilated cardiomyopathy by 
strain echocardiography. J. Am. Soc. Echocardiogr. 25:667-673. doi: 10.1016/j.echo.2012.02.004. PMID: 22421028.

Haugaa, K.H., Grenne, B.L., Eek, C.H., Ersbøll, M., Valeur, N., Svendsen, J.H., et al. $2013 a$. Strain echocardiography improves risk prediction of ventricular arrhythmias after myocardial infarction. JACC Cardiovasc. Imaging, 6(8):841-850. doi: 10.1016/j.jcmg.2013.03.005. PMID: 23850251.

Haugaa, K.H., Johnson, J.N., Bos, J.M., Phillips, B.L., Eidem, B.W., and Ackerman, M.J. $2013 b$. Subclinical cardiomyopathy and long QT syndrome: an echocardiographic observation. Congenit. Heart Dis. 8:352-359. doi: 10.1111/chd.12011. PMID: 23095322.

Hirata, K., Hyodo, E., Hozumi, T., Kita, R., Hirose, M., Sakanoue, Y., et al. 2009. Usefulness of a combination of systolic function by left ventricular ejection fraction and diastolic function by E/E' to predict prognosis in patients with heart failure. Am. J. Cardiol. 103(9):1275-1279. doi: 10.1016/j.amjcard.2009.01.024. PMID: 19406271.

Iacoviello, M., Puzzovivo, A., Guida, P., Forleo, C., Monitillo, F., Catanzaro, R., et al. 2013. Independent role of left ventricular global longitudinal strain in predicting prognosis of chronic heart failure patients. Echocardiography, 30(7):803-811. doi: 10.1111/echo.12142. PMID: 23488596.

Kalam, K., Otahal, P., and Marwick, T.H. 2014. Prognostic implications of global LV dysfunction: a systematic review and meta-analysis of global longitudinal strain and ejection fraction. Heart, 100(21):1673-1680. doi: 10.1136/heartjnl-2014-305538. PMID: 24860005.

Lang RM, Bierig M, Devereux RB, Flachskampf FA, Foster E, Pellikka PA, et al; American Society of Echocardiography's Nomenclature and Standards Committee; Task Force on Chamber Quantification; American College of Cardiology Echocardiography Committee; 
American Heart Association; European Association of Echocardiography, European Society of Cardiology. 2006. Recommendations for chamber quantification. Eur. J. Echocardiogr. 7(2):79108. doi: 10.1016/j.euje.2005.12.014. PMID: 16458610.

Khan, S.G., Klettas, D., Kapetanakis, S., and Monaghan, M.J. 2016. Clinical utility of speckletracking echocardiography in cardiac resynchronisation therapy. Echo. Res. Pract. 3(1):R1-R11. doi: 10.1530/ERP-15-0032. PMID: 27249816.

Lim, T.K., Dwivedi, G., Hayat, S., Majumdar, S., and Senior, R. 2009. Independent value of left atrial volume index for the prediction of mortality in patients with suspected heart failure referred from the community. Heart, 95(14):1172-1178. doi: 10.1136/hrt.2008.151043. PMID: 19359264.

McMurray, J.J., Adamopoulos, S., Anker, S.D., Auricchio, A., Böhm, M., Dickstein, K., et al. 2012. ESC Guidelines for the diagnosis and treatment of acute and chronic heart failure 2012: The Task Force for the Diagnosis and Treatment of Acute and Chronic Heart Failure 2012 of the European Society of Cardiology. Developed in collaboration with the Heart Failure Association (HFA) of the ESC. Eur. Heart J. 33(14):1787-1847. doi: 10.1093/eurheartj/ehs104. PMID: 22611136.

Mignot, A., Donal, E., Zaroui, A., Reant, P., Salem, A., Hamon, C., et al. 2010. Global longitudinal strain as a major predictor of cardiac events in patients with depressed left ventricular function: a multicenter study. J. Am. Soc. Echocardiogr. 23:1019-1024. doi: 10.1016/j.echo.2010.07.019. PMID: 20810243.

Mogelvang, R., Biering-Sørensen, T., and Jensen, J.S. 2015. Tissue Doppler echocardiography predicts acute myocardial infarction, heart failure, and cardiovascular death in the general 
population. Eur. Heart J. Cardiovasc. Imaging, 16(12):1331-1337. doi: 10.1093/ehjci/jev180. PMID: 26202086.

Møller, J.E., Pellikka, P.A., Hillis, G.S., and Oh, J.K. 2006. Prognostic importance of diastolic function and filling pressure in patients with acute myocardial infarction. Circulation, 114(5):438-444. doi: 10.1161/CIRCULATIONAHA.105.601005. PMID: 16880341.

Nagueh, S.F., Appleton, C.P., Gillebert, T.C., Marino, P.N., Oh, J.K., Smiseth, O.A., et al. 2009. Recommendations for the evaluation of left ventricular diastolic function by echocardiography. Eur. J. Echocardiogr. 10(2):165-193. doi: 10.1093/ejechocard/jep007. PMID: 19270053.

Nahum, J., Bensaid, A., Dussault, C., Macron, L., Clémence, D., Bouhemad, B., et al. 2010. Impact of longitudinal myocardial deformation on the prognosis of chronic heart failure patients. Circ. Cardiovasc. Imaging, 3:249-256. doi: 10.1161/CIRCIMAGING.109.910893. PMID: 20233858.

Nikitin, N.P., Loh, P.H., Silva, Rd., Ghosh, .J, Khaleva, O.Y., Goode, K., et al. 2006. Prognostic value of systolic mitral annular velocity measured with Doppler tissue imaging in patients with chronic heart failure caused by left ventricular systolic dysfunction. Heart, 92:775-779. doi: 10.1136/hrt.2005.067140. PMID: 16251233.

Olson, J.M., Samad, B.A., and Alam, M. 2008. Prognostic value of pulse-wave tissue Doppler parameters in patients with systolic heart failure. Am. J. Cardiol. 102(6):722-725. doi: 10.1016/j.amjcard.2008.04.054. PMID: 18773996.

Reisner, S.A., Lysyansky, P., Agmon, Y., Mutlak, D., Lessick, J., and Friedman, Z. 2004. Global longitudinal strain: a novel index of left ventricular systolic function. J. Am. Soc. Echocardiogr. 17(6):630-633. doi: 10.1016/j.echo.2004.02.011. PMID: 15163933. 
Rosa, I., Marini, C., Stella, S., Ancona, F., Spartera, M., Margonato, A., et al. 2016. Mechanical dyssynchrony and deformation imaging in patients with functional mitral regurgitation. World J. Cardiol. 8(2):146-62. doi: 10.4330/wjc.v8.i2.146. PMID: 26981211.

Roşca, M., Lancellotti, P., Popescu, B.A., and Piérard, L.A. 2011. Left atrial function: pathophysiology, echocardiographic assessment and clinical applications. Heart, 97:1982-1289. doi: 10.1136/heartjnl-2011-300069. PMID: 22058287.

Russo, C., Jin, Z., Elkind, M.S., Rundek, T., Homma, S., Sacco, R.L., et al. 2014. Prevalence and prognostic value of subclinical left ventricular systolic dysfunction by global longitudinal strain in a community-based cohort. Eur. J. Heart Fail. 16(12):1301-1309. doi: 10.1002/ejhf.154. PMID: 25211239.

Seo, Y., Ishizu, T., Atsumi, A., Kawamura, R., and Aonuma, K. 2014. Three-dimensional speckle tracking echocardiography. Circ. J. 78(6):1290-301. doi:10.1253/circj.CJ-14-0360. PMID: 24770358.

Smiseth, O.A., Torp, H., Opdahl, A., Haugaa, K.H., and Urheim, S. 2016. Myocardial strain imaging: how useful is it in clinical decision making? Eur. Heart J. 37(15):1196-1207. doi: 10.1093/eurheartj/ehv529. PMID: 26508168.

Stanton, T., Leano, R., and Marwick, T.H. 2009. Prediction of all-cause mortality from global longitudinal speckle strain: comparison with ejection fraction and wall motion scoring. Circ. Cardiovasc. Imaging, 2(5):356-364. doi: 10.1161/CIRCIMAGING.109.862334. PMID: 19808623.

ter Bekke, R.M., and Volders, P.G. 2012. Arrhythmogenic mechano-electric heterogeneity in the long-QT syndrome. Prog. Biophys. Mol. Biol. 110:347-358. doi: 10.1016/j.pbiomolbio.2012.07.007. PMID: 22841828. 
Wang, M., Yip, G.W., Wang, A.Y., Zhang, Y., Ho, P.Y., Tse, M.K., et al. 2003. Peak early diastolic mitral annulus velocity by tissue Doppler imaging adds independent and incremental prognostic value. J. Am. Coll. Cardiol. 41:820-826. PMID: 12628728.

Zoghbi, W.A., Enriquez-Sarano, M., Foster, E., Grayburn, P.A., Kraft, C.D., Levine, R.A., et al. 2003. Recommendations for evaluation of the severity of native valvular regurgitation with twodimensional and Doppler echocardiography. J. Am. Soc. Echocardiogr. 16(7):777-802. doi: 10.1016/S0894-7317(03)00335-3. PMID: 12835667. 
Table 1: Baseline characteristics of the study population.

Variable Data

Mean age (years)

$63 \pm 12$

Female/male gender $n(\%)$

$111(32.6 \%) / 229(67.4 \%)$

Body surface area $\left(\mathrm{m}^{2}\right)$

$1.85 \pm 0.2$

Heart rate (beats/min)

$67 \pm 13$

Mean arterial pressure $(\mathrm{mmHg})$

$106 \pm 14$

Coronary artery disease, $n(\%)$

$215(63.2)$

Non-ischemic cardiomyopathy, $n(\%)$

Systemic hypertension, $n(\%)$

New York Heart Association class I $n(\%)$

$28(8.2 \%)$

$$
\begin{aligned}
& \text { class II } n(\%) \\
& \text { class III } n(\%) \\
& \text { class IV } n(\%)
\end{aligned}
$$$$
117(34.4 \%)
$$

$17(5.0 \%)$

Medical therapy

Beta blocker, $n(\%)$

$294(86.5)$ 
ACEI/angiotensin receptor antagonist, $n(\%)$

$315(92.6)$

Diuretics, $n(\%)$

$274(77.6)$

Aldosterone antagonist, $n(\%)$

$259(76.2)$

Digoxin, $n(\%)$

$61(17.9)$

Nitrates, $n(\%)$

$231(67.9)$

Amiodarone, $n(\%)$

$32(9.4)$

Follow-up (months)

$36 \pm 9$

* Data are presented as mean $\pm \mathrm{SD}$ or absolute values $(\%)$;

Note: ACEI, angiotensin converting enzyme inhibitor. 
Table 2. Baseline echocardiographic findings in the study group.

\begin{tabular}{|c|c|c|c|}
\hline Characteristics & $\begin{array}{l}\text { Non-arrhythmic } \\
\text { events } \\
n=292 \text { ) }\end{array}$ & $\begin{array}{l}\text { Arrhythmic } \\
\text { events } \\
(n=48)\end{array}$ & $\mathrm{P}$ value \\
\hline LV end-diastolic volume (ml) & $108 \pm 34$ & $131 \pm 53$ & 0.03 \\
\hline LV end-systolic volume (ml) & $61 \pm 25$ & $87 \pm 29$ & 0.02 \\
\hline LV ejection fraction $(\%)$ & $43 \pm 12$ & $34 \pm 14$ & 0.001 \\
\hline Left atrial volume (ml) & $88 \pm 40$ & $121 \pm 51$ & 0.002 \\
\hline Systolic pulmonary artery pressure $(\mathrm{mmHg})$ & $40 \pm 13$ & $48 \pm 17$ & 0.003 \\
\hline Mitral regurgitant orifice area $\left(\mathrm{mm}^{2}\right)$ & $27 \pm 11$ & $28 \pm 8$ & 0.35 \\
\hline Mitral regurgitant volume $(\mathrm{ml})$ & $38 \pm 17$ & $42 \pm 25$ & 0.27 \\
\hline E/E' ratio & $11.6 \pm 4.4$ & $13.8 \pm 6.4$ & $<0.001$ \\
\hline $\mathrm{S}^{\prime}(\mathrm{cm} / \mathrm{s})$ & $7.5 \pm 2.9$ & $5.1 \pm 2.1$ & 0.001 \\
\hline LV GLS (\%) & $-18.1 \pm 6.5$ & $-11.1 \pm 6.5$ & $<0.001$ \\
\hline LV MD (ms) & $39.7 \pm 33.1$ & $72.3 \pm 27.6$ & $<0.001$ \\
\hline GLS/MD index $(\% / \mathrm{ms})$ & $-0.83 \pm 0.54$ & $-0.24 \pm 0.31$ & $<0.001$ \\
\hline
\end{tabular}


Note: E, early diastolic transmitral velocity; E', early diastolic mitral annular velocity; GLS, global longitudinal strain; LV, left ventricle; MD, mechanical dispersion; S', systolic mitral annular velocity. 
Table 3. Echocardiographic variables associated with major ventricular arrhythmic events in Cox univariate and multivariate analysis.

Variables

Univariate HR (CI 95\%) $\quad$ P-value $\quad$ Multivariate HR (CI 95\%) $\quad P$-value

Left atrial volume

$1.01(1.00-1.02)$

0.01

LV end-diastolic volume

$1.03(1.01-1.05)$

0.02

LV end-systolic volume

$1.08(1.02-1.13)$

0.01

LVEF

$0.94(0.92-0.97)$

$<0.001$

$0.98(0.95-1.01)$

0.23

SPAP

$1.03(1.01-1.05)$

0.01

E/E’ ratio

$1.21(1.15-1.27)$

$<0.001$

$1.14(1.08-1.22)$

0.008

S' velocity

$0.63(0.52-0.75) \quad 0.001$

GLS

$1.16(1.11-1.22)$

$<0.001$

$1.01(0.93-1.09)$

0.91

MD

$1.02(1.01-1.03)$

$<0.001$

$1.00(0.97-1.02)$

0.13

GLS/MD

$2.59(1.35-4.95)$

$<0.001$

$3.22(1.72-6.15)$

0.03

Note: CI, confidence interval; E, early diastolic transmitral velocity; E', early diastolic mitral annular velocity; GLS, global longitudinal strain; HR, hazard ratio; LVEF, left ventricular ejection fraction; MD, mechanical dispersion; S', systolic mitral annular velocity; SPAP, systolic pulmonary artery pressure. 
Table 4. Echocardiographic findings in 217 patients with LVEF $>35 \%$ and 123 patients with LVEF $\leq 35 \%$.

\begin{tabular}{lrr}
\hline \hline Characteristics & Patients without & Patients with $P$ value \\
& arrhythmic events & arrhythmic events \\
& \\
\hline
\end{tabular}

Patients with LVEF $>35 \%$

\begin{tabular}{|c|c|c|c|}
\hline LVEF (\%) & $48.9 \pm 9.5$ & $47 \pm 8.1$ & 0.21 \\
\hline $\mathrm{E} / \mathrm{E}^{\prime}$ ratio & $10.6 \pm 4.1$ & $18.8 \pm 6.9$ & 0.001 \\
\hline $\mathrm{S}^{\prime}(\mathrm{cm} / \mathrm{s})$ & $9.3 \pm 2.9$ & $7.5 \pm 1.9$ & 0.004 \\
\hline LV GLS (\%) & $-18.8 \pm 6.1$ & $-13.7 \pm 8.1$ & 0.001 \\
\hline LV MD (ms) & $31.2 \pm 25.2$ & $63.1 \pm 29.2$ & $<0.001$ \\
\hline GLS/MD index $(\% / \mathrm{ms})$ & $-0.99 \pm 0.52$ & $-0.37 \pm 0.41$ & $<0.001$ \\
\hline
\end{tabular}

Patients with $L$ VEF $\leq 35 \%$

\begin{tabular}{lccc} 
LVEF $(\%)$ & $29.9 \pm 5.2$ & $23.4 \pm 6.9$ & $<0.001$ \\
E/E' ratio & $13.6 \pm 4.4$ & $17.1 \pm 6.1$ & 0.001 \\
$\mathrm{~S}^{\prime}(\mathrm{cm} / \mathrm{s})$ & $3.78 \pm 0.5$ & $2.99 \pm 0.3$ & 0.002 \\
\hline
\end{tabular}




\begin{tabular}{lccc}
\hline LV GLS (\%) & $-14.2 \pm 5.6$ & $-8.9 \pm 3.5$ & $<0.001$ \\
LV MD (ms) & $56.9 \pm 39.5$ & $80.2 \pm 24.1$ & 0.005 \\
GLS/MD index $(\% / \mathrm{ms})$ & $-0.43 \pm 0.04$ & $-0.11 \pm 0.02$ & $<0.001$ \\
\hline
\end{tabular}

Note: E, early diastolic transmitral velocity; E', early diastolic mitral annular velocity; EF, ejection fraction; GLS, global longitudinal strain; LV, left ventricle; MD, mechanical dispersion; S', systolic mitral annular velocity. 


\section{Figure captions}

Figure 1. Representative example of the calculation of GLS/MD ratio. MD was calculated as the standard deviation of time to peak regional negative strain. GLS/MD was determined ($19.4 \% / 39.3 \mathrm{~ms}=-0.49 \% / \mathrm{ms})$

AVC, aortic valve closure; ANT, anterior; GLS, global longitudinal strain; INF, inferior; LAT, lateral; MD, mechanical dispersion; POST, posterior; SEPT, septal.

Figure 2. The additional benefit of GLS/MD ratio to predict arrhythmic events. The addition of GLS/MD ratio markedly improved the prognostic utility of the model containing LVEF, S' wave, E/E' ratio, GLS and $\mathrm{MD}(p=0.001)$.

E, early diastolic transmitral velocity; E', early diastolic mitral annular velocity; GLS, global longitudinal strain; LVEF, left ventricular ejection fraction; MD, mechanical dispersion; S', systolic mitral annular velocity.

$* p<0.05$

Figure 3. The receiver-operator characteristic (ROC) curve for GLS/MD ratio to predict major ventricular arrhythmic events.

AUC, area under ROC curve; GLS, global longitudinal strain; MD, mechanical dispersion; 95\% CI, $95 \%$ confidence interval. 
Figure 4. Kaplan-Meier survival curves in the overall population (340 patients) with heart failure (a), in those with left ventricular ejection fraction (LVEF) $\leq 35 \%$ (b), and in those with LVEF $>35 \%$ (c), according to GLS/MD ratio below and above $-0.2 \% / \mathrm{ms}$.

GLS, global longitudinal strain; MD, mechanical dispersion. 


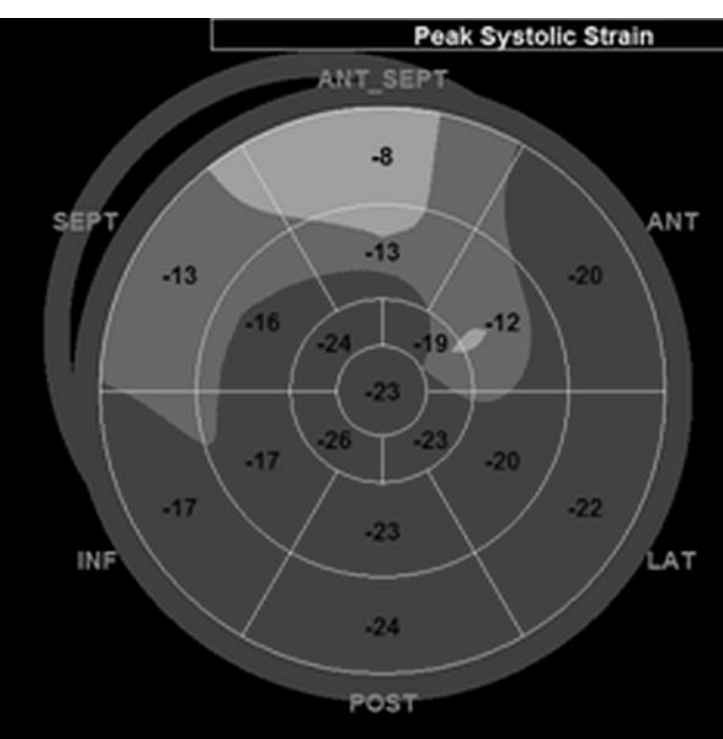

V
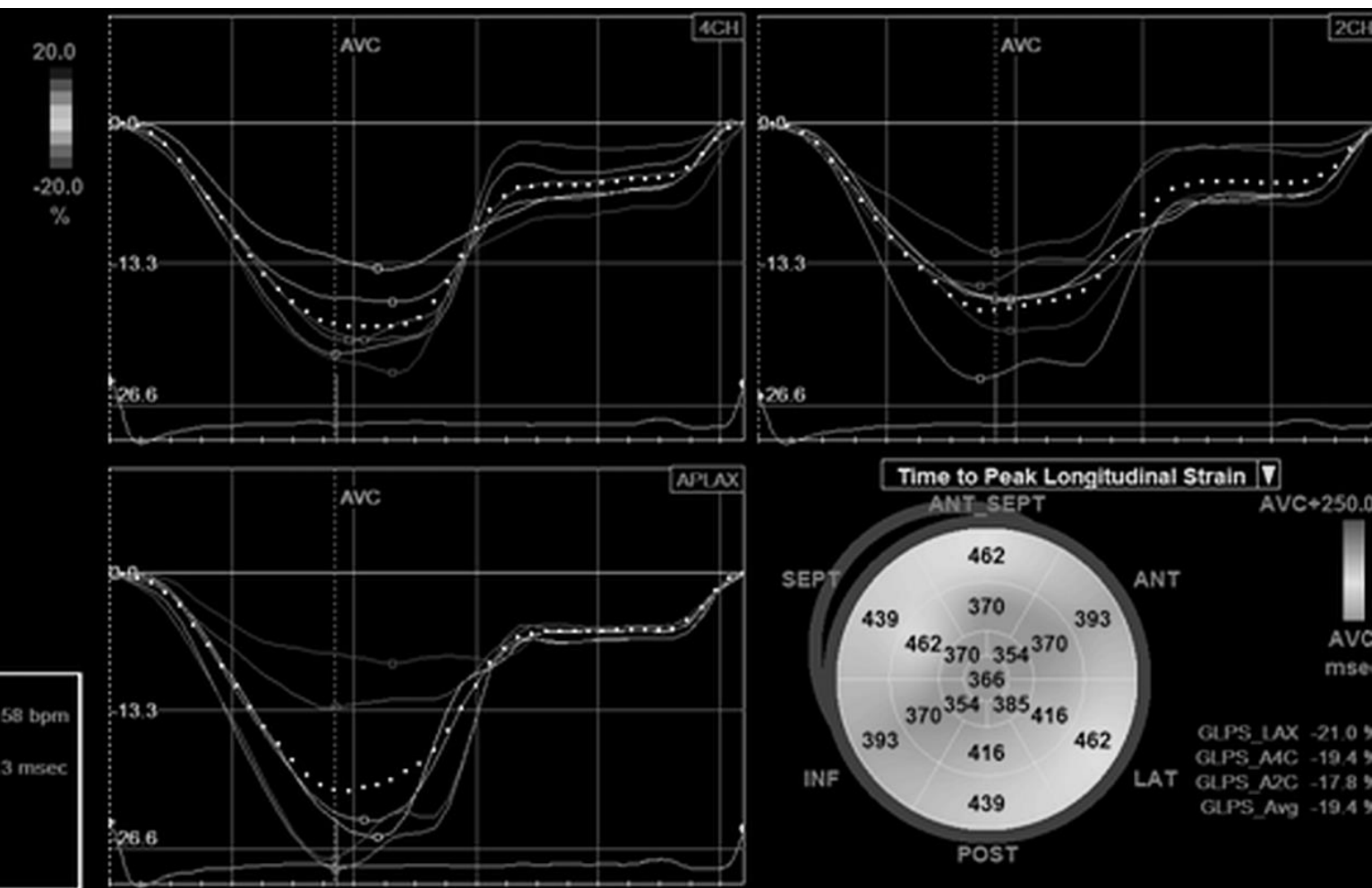

https://mc06.manuscriptcentral.com/cjpp-pubs 


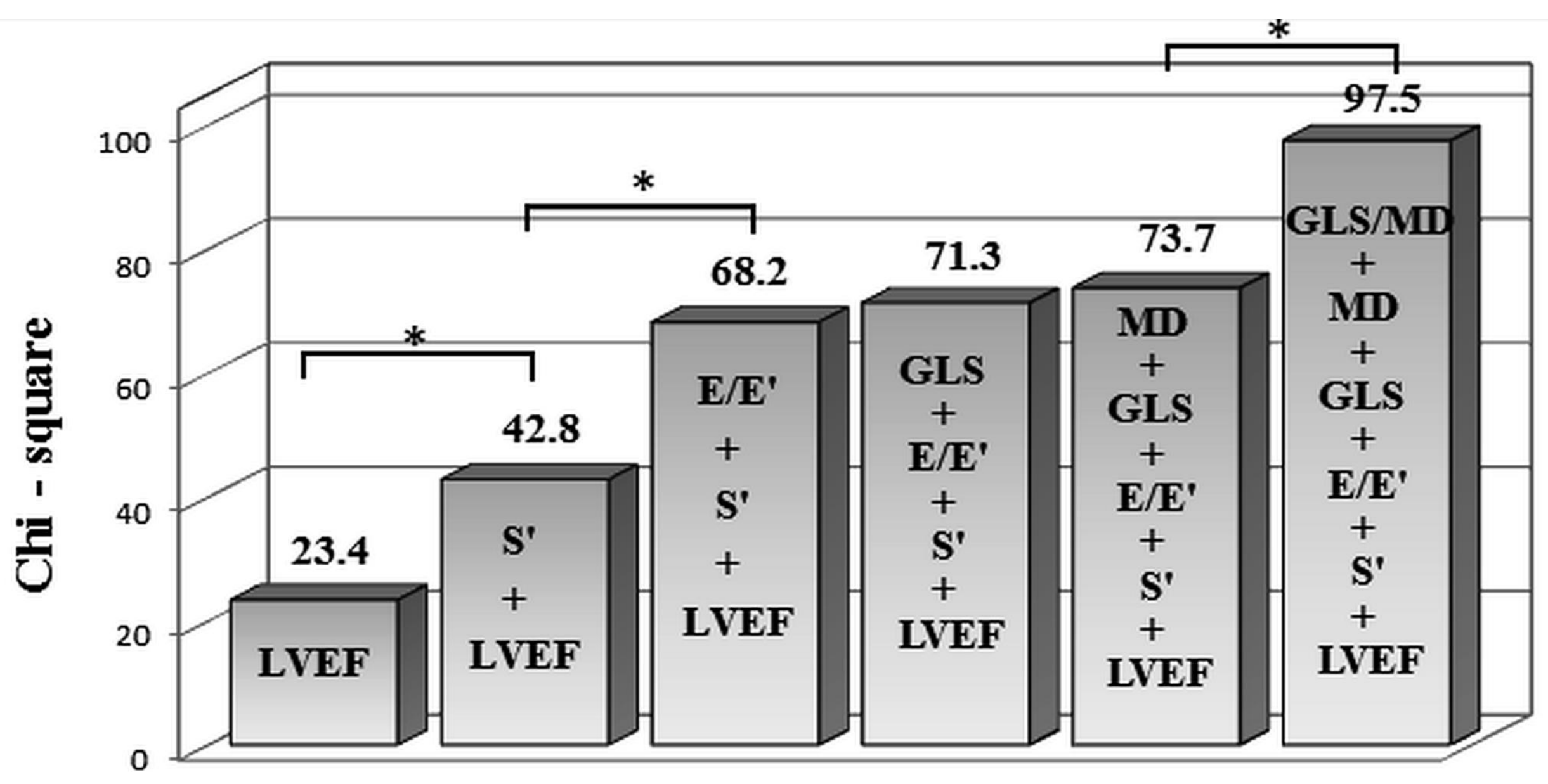



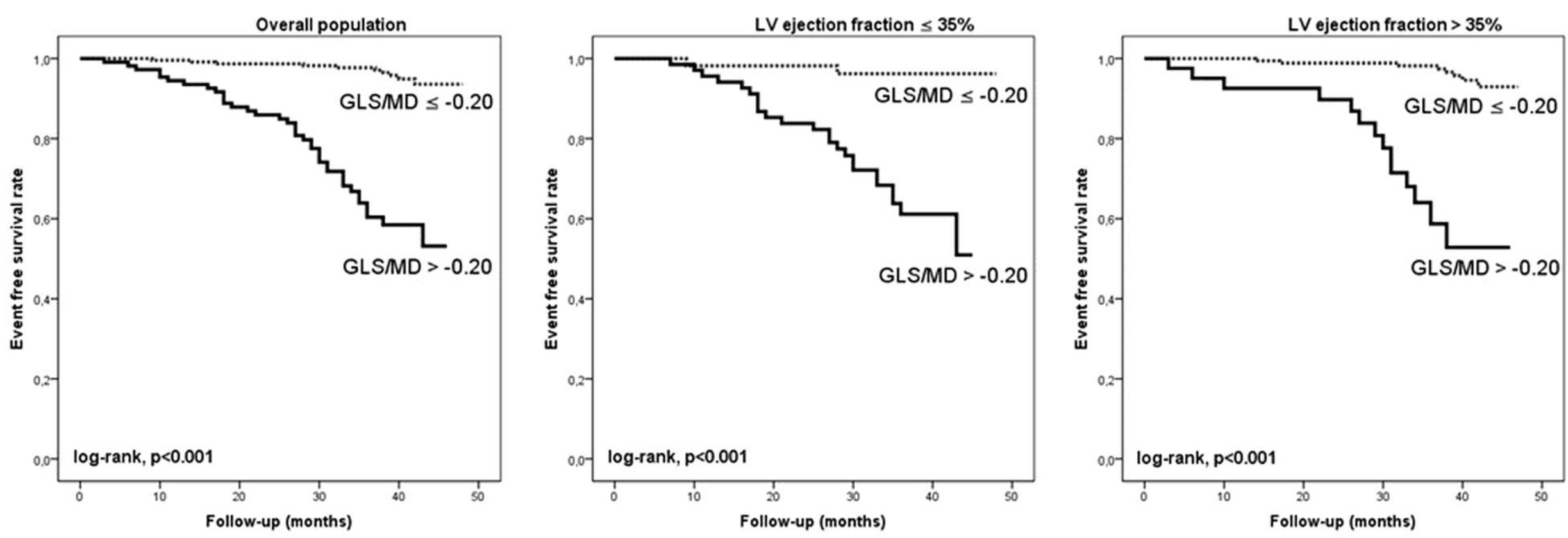


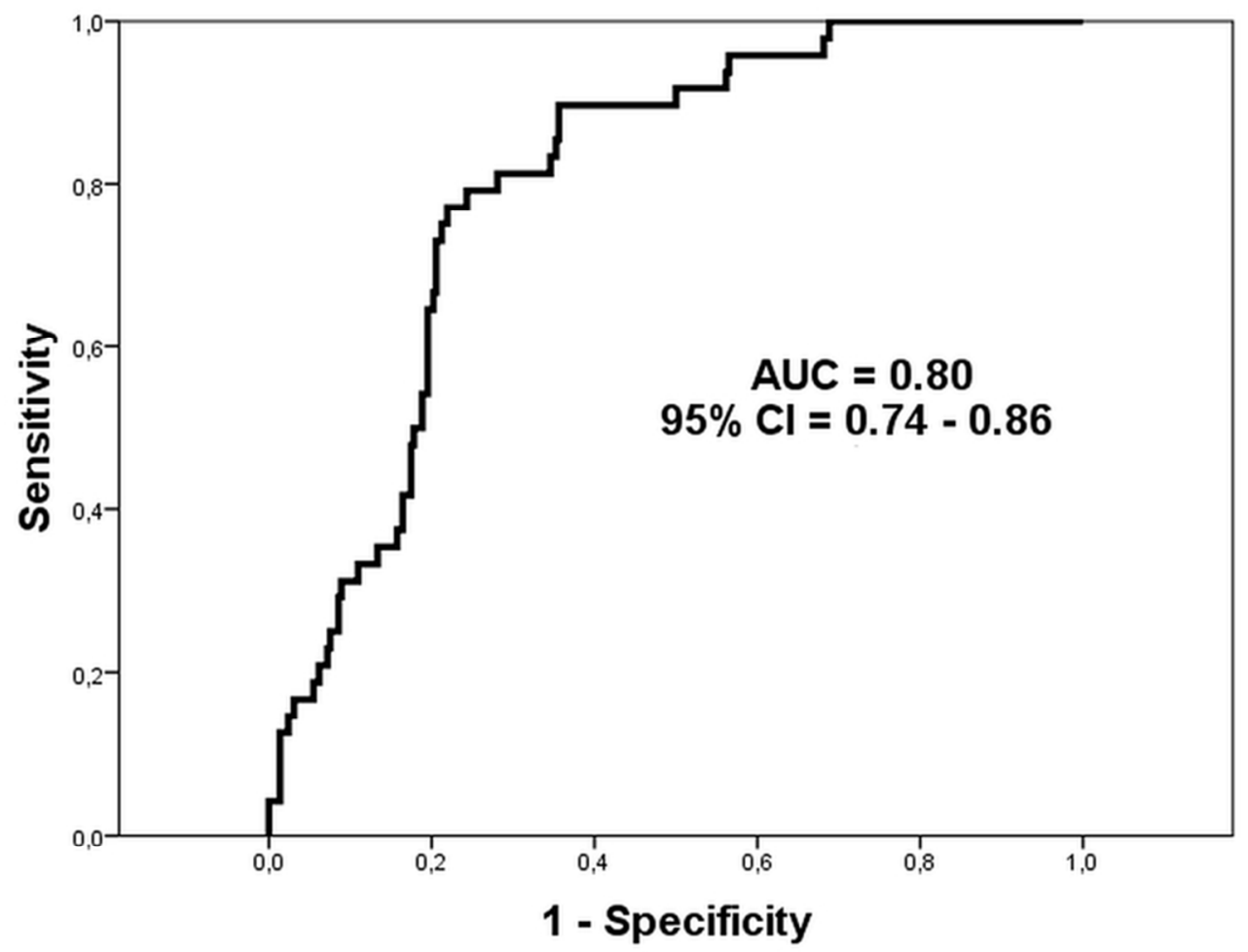

Figure 3. The receiver-operator characteristic (ROC) curve for GLS/MD ratio to predict major ventricular arrhythmic events.

AUC, area under ROC curve; GLS, global longitudinal strain; MD, mechanical dispersion; $95 \%$ CI, 95\% confidence interval.

$68 \times 55 \mathrm{~mm}(300 \times 300 \mathrm{DPI})$ 